\title{
Glutamine synthetase from Lactobacillus hilgardif LMG 7934
}

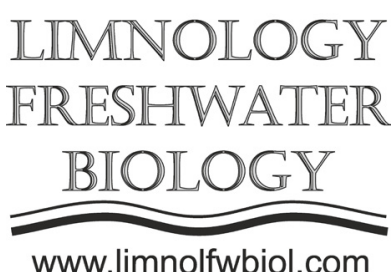

\author{
Zhuravleva D.E.*, Yadykova L.L., Iskhakova Z.I., Kayumov A.R. \\ Kazan Federal University, Kremlevskaya Str., 18, Kazan, 420008, Russia
}

\begin{abstract}
While lactic acid bacteria are widespread in various ecological niches with the ex cess of nutrients and extensive use in food industry, many aspects of the nitrogen metabolism of Lactobacilli remain unexplored. The most preferred nitrogen sources for most bacteria are glutamine and ammonium ions. In bacterial cells an enzyme glutamine synthetase synthetizes glutamine from glutamate and ammonium ion. While L. hilgardii LMG 7934 genome carries two genes encoding glutamine synthetases with $55 \%$ of mutual identity of amino acid sequences, which protein plays the major role is still unclear. One gene is located within classical $g \ln R A$ operon with the gene of GlnR-like transcriptional regulator, while the second is monocistronic. In this study we report the cloning of both genes encoding glutamine synthetases from L. hilgardii LMG 7934.
\end{abstract}

Keywords: Nitrogen metabolism, Lactobacilli, glutamine synthetase.

\section{Introduction}

Lactobacillus are Gram-positive, rod-shaped, facultative aerobic or microaerophilic, non-spore forming bacteria (Makarova et al., 2006). Lactic acid bacteria are widespread in nature and can be found in niches with the excess of nutrients, while could be almost never found neither in soil nor water. Despite extensive use in food industry, in production of probiotics, lactic acid and silage, etc. (De Vos et al., 2009), many aspects of the nitrogen metabolism of Lactobacilli remain unexplored. Since the most bacteria are not capable of the biological nitrogen fixation, the preferred nitrogen sources for them are glutamine and ammonium ions which could be directly involved to the nitrogen metabolism of the cell (Hu et al., 1999). Glutamine synthetase (GS) is a metalloenzyme catalyzing the ATP-dependent synthesis of glutamine from glutamate and ammonium (Wray and Fisher, 2005). This enzyme is involved in GS/ GOGAT ammonium assimilation cycle, which is active in conditions of low concentrations of ammonium ions. Glutamate synthase (GOGAT) synthesizes two glutamic acid molecules from glutamine and 2-oxoglutarate. One molecule is consumed for the cell needs, and the second is converted to glutamine by glutamine synthetase in the presence of an ammonium ion (Van Heeswijk et al., 2013). While lactic acid bacteria are widespread in various ecological niches with the excess of nutrients, the presence of gene encoding glutamine synthetase in their genome suggests the fundamental role of this enzyme for the cell.

\section{Materials and methods}

The Lactobacillus hilgardii LMG 7934 (ATCC 27305) strain from Belgian Coordinated Collections of Microorganisms (BCCM) was used in this study. For the DNA extraction a single colony of $L$. hilgardii LMG 7934 was grown in Man-Rogosa-Sharpe (MRS) Broth (SigmaAldrich, USA) under microaerophilic conditions at $37^{\circ} \mathrm{C}$ overnight. The genomic DNA was extracted using GeneJET Genomic DNA Purification Kit (ThermoFisher, USA).The quality and purify of DNA was checked by using $0.7 \%$ agarose gel electrophoresis, while the quantity was determined using Nanodrop2000 systems. The $g \ln A$ genes was cloned into expression vectors used Gibson`s reaction. The GS proteins were purified using affinity chromatography on Ni-NTA sepharose. The GS enzymatic activity was determined by biosynthetic assay (Patterson and Hespell, 1985; Fedorova et al., 2013).

\section{Results and discussion}

In $L$. hilgardii genome, two glutamine synthetase genes are present, one is located in the glnRA operon with the transcriptional factor GlnR gene $(g \ln R)$, and the second gene is monocistronic. Both genes were cloned onto pET15b expression vector, resulting GS1-his and GS2-his recombinant proteins were purified to an electrophoretic homogeneity. To test the enzymatic activity of GS in vitro and in vivo assays was performed. Both purified proteins showed a low level 
of biosynthetic activity in vitro in compare with the GS activity in vivo in Lactobacillus cells.

\section{Acknowledgments}

This research was supported by Council on grants of the President of the Russian Federation (MD572.2020.4 for AK) and performed in frames of Russian Government Program of Competitive Development of Kazan Federal University.

\section{References}

De Vos P., Garrity G.M., Jones D. et al. 2009. Bergey's manual of systematic bacteriology, 2nd edition, Volume 3: The Firmicutes. New York: Springer-Verlag. DOI: 10.1007/978-0-387-68489-5

Fedorova K., Kayumov A., Woyda K. et al. 2013. Transcription factor TnrA inhibits the biosynthetic activity of glutamine synthetase in Bacillus subtilis. FEBS Letters 587: 1293-1298. DOI: 10.1016/j.febslet.2013.03.015
Hu P., Leighton T., Ishkhanova G. et al. 1999. Sensing of nitrogen limitation by Bacillus subtilis: comparison to enteric bacteria. Journal of Bacteriology 181: 5042-5050. DOI: 10.1128/JB.181.16.5042-5050.1999

Makarova K., Slesarev A., Wolf Y. et al. 2006. Comparative genomics of the lactic acid bacteria. Proceedings of the National Academy of Sciences of the United States of America 103: 15611-15616. DOI: $10.1073 /$ pnas.0607117103

Patterson J.A., Hespell R.B. 1985. Glutamine synthetase activity in the ruminal bacterium Succinivibrio dextrinosolvens. Applied and Environmental Microbiology 50: 1014-1020.

Van Heeswijk W.C., Westerhoff H.V., Boogerd F.C. 2013. Nitrogen assimilation in Escherichia coli: putting molecular data into a systems perspective. Microbiology and Molecular Biology Reviews 77: 628-695. DOI: 10.1128/MMBR.00025-13

Wray L.V., Fisher S.H. 2005. A feedback-resistant mutant of Bacillus subtilis glutamine synthetase with pleiotropic defects in nitrogen-regulated gene expression. The Journal of Biological Chemistry 280: 33298-33304. DOI: $10.1074 / \mathrm{jbc}$. M504957200 